\title{
A multi-objective particle swarm optimization for production-distribution planning in supply chain network
}

\author{
Alireza Pourrousta $^{\mathrm{a}}$, Saleh dehbari ${ }^{\mathrm{a}^{*}}$, Reza Tavakkoli-Moghaddam ${ }^{\mathrm{b}}$ and Mohsen sadegh amalnik ${ }^{\mathrm{b}}$
}

${ }^{a}$ Department of Industrial Engineering, Islamic Azad University, South Tehran Branch, Iran

${ }^{b}$ Department of Industrial Engineering, College of Engineering, University of Tehran, Tehran, Iran

\begin{tabular}{l}
\hline A R T I C L E I N F O \\
\hline Article history: \\
Received October 2, 2011 \\
Received in Revised form \\
November, 15, 2011 \\
Accepted 20 November 2011 \\
Available online \\
25 November 2011 \\
\hline Keywords: \\
Multi-objective optimization \\
Ranking fuzzy numbers \\
Multi-objective particle swarm \\
optimization \\
NSGA-II
\end{tabular}

\section{A B S T R A C T}

\begin{abstract}
Integrated supply chain includes different components of order, production and distribution and it plays an important role on reducing the cost of manufacturing system. In this paper, an integrated supply chain in a form of multi-objective decision-making problem is presented. The proposed model of this paper considers different parameters with uncertainty using trapezoid numbers. We first implement a ranking method to covert the fuzzy model into a crisp one and using multi-objective particle swarm optimization, we solve the resulted model. The results are compared with the performance of NSGA-II for some randomly generated problems and the preliminary results indicate that the proposed model of the paper performs better than the alternative method.
\end{abstract}

(c) 2012 Growing Science Ltd. All rights reserved.

\section{Introduction}

During the past few years, there have been significant attempts for providing integrated supply chain problems, which includes suppliers, manufacturers, distributors and retailers. The primary objective of an integrated supply chain (SC) is to optimize all cost components from converting raw materials into final products delivered to end users (Davis, 1993; McDonald \& Karimi, 1997; Simchi-Levi et al., 2000). Sabri and Beamon (2000) developed a comprehensive multi-objective SC to implement in simultaneous strategic and operational SC planning. The adapted multi-objective decision analysis allows us to use a performance measurement system, which includes cost, customer service levels (fill rates), and flexibility.

Syarif et al. (2002) developed a new logistic chain network problem, which is a $0-1$ mixed integer linear programming model. The design tasks of this problem considered the choice of the facilities to be opened and the distribution network design to satisfy the demand with minimum cost. They used the spanning tree-based genetic algorithm by implementing Prüfer number representation. The

\footnotetext{
* Corresponding author. Tel: +989369427662

E-mail addresses: dehbari@gmail.com (S. Dehbari) 
efficiency of the proposed method was examined by comparing its numerical experiment results with those of conventional matrix-based genetic algorithm. Zhou et al. (2002) implemented a genetic algorithm for a balanced allocation of customers to multiple distribution centers in the supply chain network.

Jayaraman and Ross (2003) addressed a system of distribution network design problems characterized by multiple product families, a central manufacturing plant site, multiple distribution center and cross-docking sites, and retail outlets (customer zones) which demand multiple units of various commodities.

Dubois et al. (2003) studied different fuzzy set-based approaches for scheduling which includes representing preference profiles and modeling uncertainty distributions. Chen and Chang (2006) presented a mathematical programming method for supply chain models with fuzzy parameters. Liang (2008) presented an integrated production-transportation planning decision with fuzzy multiple objectives in supply chains. Aliev et al. (2007) used fuzzy-genetic approach to aggregate productiondistribution planning in supply chain management. Abido (2007) presented a two-level nondominated solutions approach for multi-objective particle swarm optimization.

Liang (2008) presented a fuzzy multi-objective production/distribution planning decisions with multiproduct and multi-time period in a supply chain. Liang extended a fuzzy multi-objective linear programming (FMOLP) system with piecewise linear membership function to handle integrated multi-product and multi-time period production/distribution planning decisions (PDPD) problems where the objectives are formulated in fuzzy form.

The work extends the original multi-objective linear programming to minimize total costs and total delivery time associated with inventory levels, available machine capacity and labor levels at each source, and predicts demand and available warehouse space at each destination and total budget. The proposed FMOLP model presents a systematic framework, which facilitates fuzzy decision-making process to adjust the search direction during the solution procedure to obtain a DM's efficient solution. In addition, the DM calculates the value in each cost category by studying the time value of money in the proposed model.

Peidro et al. (2009) developed a fuzzy mathematical programming model for supply chain planning, which studies supply, demand and process uncertainties. The model was formulated as a fuzzy mixed-integer linear programming model, where data were ill-known and modeled by triangular fuzzy numbers. Peidro et al. (2010), in another work, studied a fuzzy linear programming based method for tactical supply chain planning in an uncertainty environment. Torabi and Hassini (2008) presented an interactive possibilistic programming method for multiple objective supply chain master planning and their computational results indicated that the proposed fuzzy method relatively performed better than other fuzzy techniques.

Bilgen (2010) developed an integration of production and distribution system into a unified model and addressed the production and distribution planning problem in a supply chain system, which includes the allocation of production volumes among various production lines in the manufacturing plants, and the delivery of the goods to the distribution centers. The proposed model was transformed into fuzzy models, which considers the fuzziness in the capacity constraints, and the aspiration level of costs based on various aggregation operators.

Mula et al. (2010) examined the effectiveness of a fuzzy mathematical programming system for supply chain production planning with fuzzy demand. The work incorporated a method of possibilistic programming, which makes it possible to model the epistemic uncertainty in demand, which could exist in the supply chain production planning problems as triangular fuzzy numbers. 
Dehbari et al. (2012) presented a supply chain problem where a whole seller/producer distributes goods among various retailers. The model was formulated as a more general and realistic form of traditional vehicle routing problem (VRP). The problem was solved using a hybrid of particle swarm optimization and simulated annealing (PSO-SA) and the results were compared with other hybrid method, which was a hybrid of Ant colony and Tabu search. They implemented some well-known benchmark problems to compare the results of the proposed model with other method.

Liang and Cheng (2009) applied fuzzy sets for an integrated manufacturing/distribution planning decision (MDPD) problems with multi-product and multi-time period in supply chains. The proposed model considered time value of money for each of the operating cost categories and using fuzzy multi-objective linear programming model (FMOLP) minimizes total costs and total delivery time with reference to inventory levels, available machine capacity and other issues, simultaneously. They used an industrial case to demonstrate the feasibility of the proposed model for a realistic MDPD problem.

In this paper, we present an integrated supply chain in a form of multi-objective decision-making problem. The proposed model of this paper considers different parameters with uncertainty using trapezoid numbers. We first implement a ranking method to covert the fuzzy model into a crisp one and using multi-objective particle swarm optimization, we solve the resulted model. The results are compared with the performance of NSGA-II for some benchmark problems and it indicates that the proposed model of the paper performs better than the alternative method. The organization of this paper first presents the necessary notations and problem formulations in section 2 and the metaheuristic method is given in section 3, finally, the paper concludes the results and suggests some future works.

\section{Problem statement}

\subsection{Problem definition}

The proposed model of this paper consists of four different stages. In the first stage, the SC considers $S$ suppliers providing raw material and work in process for different factories. The second stage considers $P$ factories, which produce the final product. In the third stage, the network includes $W$ distribution centers, which are responsible for shipping final products to different locations. Finally, the last stage includes $Z$ sales zones. The following assumptions hold for the proposed model of this paper,

- The supply chain includes suppliers, factories, distributers and sales centers,

- There are four cost items including purchasing, production, transportation, setup and holding,

- The input data are inventory capacity, production capacity, consumption rate, demand and supply,

- The outputs are appropriate program for purchasing, production of each factory in each period, optimal inventory level in factories and distribution centers, the amount of raw material shipped from supplier to factory and from factory to distribution center and from distribution center to end customer via sales' centers.

We consider a medium term planning and all parameters are in trapezoid fuzzy numbers. Table 1 shows the necessary parameters and decision variables, 
Table 1

Necessary notations and decision variables

\begin{tabular}{ll}
\hline Set of indices & \\
\hline$S$ & set of suppliers $(s=1,2, \ldots, S)$ \\
$P$ & set of plants $(p=1,2, \ldots, P)$ \\
$W$ & set of distribution centers $(\mathrm{DC})(w=1,2, \ldots, W)$ \\
$Z$ & set of customer zones $(\mathrm{CZ})(z=1,2, \ldots, Z)$ \\
$T$ & set of time periods $(t=1,2, \ldots, T)$ \\
$R$ & set of raw materials $(r=1,2, \ldots, R)$ \\
$G$ & set of finished products $(g=1,2, \ldots, G)$
\end{tabular}

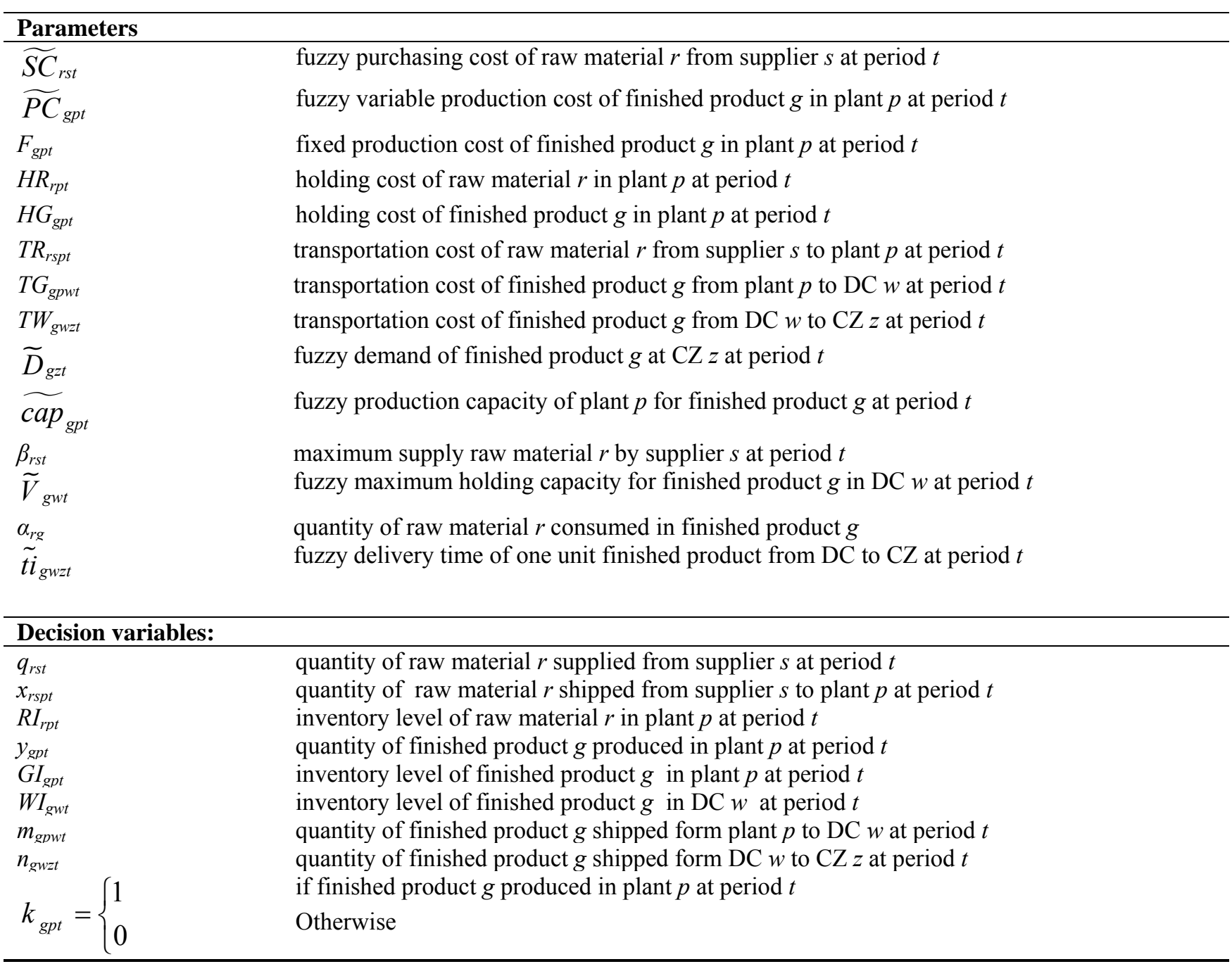

\subsection{Problems formulation}

The first objective function of the proposed model given in Eq. (1) minimizes total cost of purchasing items, setup of each product in each factory, production, inventory cost items including the cost of raw material, final product in factory and distribution centers. The objective function of the proposed model also minimizes transportation cost of raw material, final product in factory and distribution centers. The second objective function given in Eq. (2) minimizes delivery times of products for customers. 
$\min Z_{1}=\sum_{r} \sum_{s} \sum_{t} \widetilde{S C}_{r s t} q_{r s t}+\sum_{g} \sum_{p} \sum_{t}\left(F_{g p t} z_{g p t}+\widetilde{P C}_{g p t} y_{g p t}+H G_{g p t} G I_{g p t}\right)+\sum_{r} \sum_{s} \sum_{p} \sum_{t} T R_{r s p t} x_{r s p t}$

$+\sum_{g} \sum_{p} \sum_{w} \sum_{t} T G_{g p w t} m_{g p w t}+\sum_{g} \sum_{w} \sum_{z} \sum_{t} T W_{g w z t} n_{g w z t}+\sum_{r} \sum_{p} \sum_{t} H R_{r p t} R I_{r p t}+\sum_{g} \sum_{w} \sum_{t} H W_{g w t} W I_{g w t}$

$\operatorname{Min} Z_{2}=\sum_{g} \sum_{w} \sum_{z} \sum_{t} \widetilde{t i}_{g w z t} n_{g w z t}$

subject to

$$
\begin{array}{ll}
q_{r s t} \geq \sum_{p} x_{r s p t} & \forall r, s, t \\
R I_{r p t}=R I_{r p, t-1}+\sum_{s} x_{r s p t}-\sum_{g} \alpha_{r g} \cdot y_{g p t} & \forall r, p, t \\
G I_{g p t}=G I_{g p, t-1}+y_{g p t}-\sum_{w} m_{g p w t} & \forall g, p, t \\
W I_{g w t}=W I_{g w, t-1}+\sum_{p} m_{g p w t}-\sum_{z} n_{g w z t} & \forall g, w, t \\
\widetilde{D}_{g z t} \leq \sum_{w} n_{g w z t} & \forall g, z, t \\
y_{g p t} \leq \widetilde{c a p}_{g p t} k_{g p t} & \forall g, p, t \\
\sum_{p} m_{g p w t} \leq \widetilde{V}_{g w t} & \forall g, w, t \\
q_{r s t} \leq \beta_{r s t} & \forall r, s, t \\
\sum_{w} m_{g p w t} \leq M_{g p t} k_{g p t} & \forall g, p, t \\
q_{r s t}, x_{r s p t}, R I_{r p t}, y_{g p t}, G I_{g p t}, W I_{g w t}, m_{g p w t}, n_{g w z t}, B_{g w t} \geq 0, k_{g p t} \in\{0,1\} & \forall r, s, p, t, g, w, z
\end{array}
$$

Constraints (3) ensures that the amount of supplied raw material is, at least, equal to the amount of raw material shipped to all factories. Eq. (4) shows that the amount of raw material in each period is equal to the amount of inventory in the previous period and the amount of raw material shipped to factory in this period minus the consumption in this period. Eq. (5) and Eq. (6) do similarly for production and distribution centers. Eq. (7) determines the maximum demand for each distribution center. Eq. (8) and Eq. (9) show the maximum production capacity of each production and distribution centers, respectively. Eq. (10) determines the maximum supply and Eq. (11) ensures that when product is about to be delivered, the production must be setup and accomplished. Finally, Eq. (12) ensures the non-negativity of variables.

\subsection{Fuzzy model}

In this section, we present a fuzzy approach to handle the uncertainty and implement the ranking method introduced by Yao and $\mathrm{Wu}(2000)$ to defuzzify the fuzzy numbers. The distance for two fuzzy numbers $\widetilde{D}, \widetilde{E}$ is defined as follows,

$d(\widetilde{D}, \widetilde{E})=\frac{1}{2} \int_{0}^{1}\left[D_{L}(\alpha)+D_{R}(\alpha)-E_{L}(\alpha)-E_{R}(\alpha)\right] d \alpha$, 
where $D_{L}(\alpha), D_{R}(\alpha)$ are the left and right sides of fuzzy numbers with $\alpha$-cut. A trapezoid number $\widetilde{A}=\left\{a_{1}, a_{2}, a_{3}, a_{4}\right\}$ is defined as follows,

$d(\widetilde{A}, 0)=\frac{1}{2} \int_{0}^{1}\left[a_{1}+\left(a_{2}-a_{1}\right) \alpha+a_{4}-\left(a_{4}-a_{3}\right) \alpha\right] d \alpha=\frac{1}{4}\left(a_{1}+a_{2}+a_{3}+a_{4}\right)$.

Let $\widetilde{A}, \widetilde{B}$ be two fuzzy numbers, the ranking is defined as follows,

$\widetilde{A} \leq \widetilde{B} \Leftrightarrow d(\widetilde{A}, 0) \leq d(\widetilde{B}, 0)$

The mathematical model given in Eq. (1) to Eq. (12) in fuzzy form is as follows,

$$
\begin{aligned}
\min Z_{1} & =\sum_{r} \sum_{s} \sum_{t} \frac{1}{4}\left(S C_{r s t}^{1}+S C_{r s t}^{2}+S C_{r s t}^{3}+S C_{r s t}^{4}\right) q_{r s t} \\
& +\sum_{g} \sum_{p} \sum_{t}\left(F_{g p t} z_{g p t}+\frac{1}{4}\left(P C_{g p t}^{1}+P C_{g p t}^{2}+P C_{g p t}^{3}+P C_{g p t}^{4}\right) y_{g p t}+H G_{g p t} G I_{g p t}\right) \\
& +\sum_{r} \sum_{s} \sum_{p} \sum_{t} T R_{r s p t} x_{r s p t}+\sum_{g} \sum_{p} \sum_{w} \sum_{t} T G_{g p w t} m_{g p w t}+\sum_{g} \sum_{w} \sum_{z} \sum_{t} T W_{g w z t} n_{g w z t} \\
& +\sum_{r} \sum_{p} \sum_{t} H R_{r p t} R I_{r p t}+\sum_{g} \sum_{w} \sum_{t} H W_{g w t} W I_{g w t}
\end{aligned}
$$

$\min Z_{2}=\sum_{g} \sum_{w} \sum_{z} \sum_{t} \frac{1}{4}\left(t i_{g w z t}^{1}+t i_{g w z t}^{2}+t i_{g w z t}^{3}+t i_{g w z t}^{4}\right) n_{g w z t}$

subject to

$$
\begin{array}{ll}
\frac{1}{4}\left(D_{g z t}^{1}+D_{g z t}^{2}+D_{g z t}^{3}+D_{g z t}^{4}\right) \leq \sum_{w} n_{g w z t} & \forall g, z, t \\
y_{g p t} \leq \frac{1}{4}\left(c a p_{g p t}^{1}+c a p_{g p t}^{2}+c a p_{g p t}^{3}+c a p_{g p t}^{4}\right) k_{g p t} & \forall g, p, t \\
\sum_{p} m_{g p w t} \leq \frac{1}{4}\left(V_{g w t}^{1}+V_{g w t}^{2}+V_{g w t}^{3}+V_{g w t}^{4}\right) & \forall g, w, t
\end{array}
$$

The other equations are the same as the crisp model.

\section{Multi objective particle swarm optimization (MOPSO)}

Kennedy and Eberhart (1995) are believed the first who introduced particle swarm optimization (PSO). The algorithm intuitively uses how fishes and birds behave in practice, which is moving as a group in one direction and close to each other. This happens by exchanging information among members, it starts with some initial solutions, and the quality of solutions is improved to reach better solutions. In this algorithm, pbest is the best local solution and gbest is the global best solution, which are chosen from local and global particles. In each iteration, position and speed of each particle are calculated as follows,

$v(k+1)=\chi\left[\omega(k) v(k)+c_{1} r_{1}[p(k)-x(k)]+c_{2} r_{2}[g(k)-x(k)]\right]$, 
$x(k+1)=x(k)+v(k+1)$,

where $v$ is the speed of particle, $x$ is the position of each particle, $p$ and $g$ are pbest and gbest, respectively, $\chi$ and $\omega$ are contraction and inertia weights, respectively, $c_{2}$ and $c_{1}$ factors representing personal and social characteristics and we normally assign a value 2 for both and $r_{1}$ and $r_{2}$ are random numbers in interval of $[0,1]$. The first multi-objective PSO was proposed by Moore and Chapman (1999) and Coello Coello and Lechuga (2002) extended the method by introducing the following,

$v(k+1)=\chi\left[\omega(k) v(k)+c_{1} r_{1}[p(k)-x(k)]+c_{2} r_{2}[\operatorname{rep}(h)-x(k)]\right]$.

In this method, rep keeps all non-dominated solutions achieved so far.

\subsection{MOPSO implementation}

The following summarizes the implementation MOPSO.

Step 1: Set the time counter $t=0$ and generate randomly $\mathrm{n}$ particles, $\{X j(0), j=1, \ldots, n\}$, where $X j(0)=[x j, 1(0), \ldots, x j, m(0)] \cdot x j, k(0)$ is generated by randomly choosing a value with uniform probability over the $k^{\text {th }}$ best parameter search space $\left[x_{\min }^{k}, x_{\max }^{k}\right]$, Similarly, produce randomly initial velocities of all particles, $\{V j(0), j=1, \ldots, n\}$, where $V_{j}(0)=\left[v_{j, l}(0), \ldots, v_{j, m}(0)\right] . v_{j, k}(0)$ is produced by randomly choosing a value with uniform probability over the $k^{\text {th }}$ dimension $\left[v_{\min }^{k}, v_{\max }^{k}\right]$. Each particle in the initial population is updated using the objective problems. For each particle, set $S_{j}^{*}(0)$ $=\left\{X_{j}(0)\right\}$ and the local best $X_{j}^{*}(0)=X_{j}(0), j=1, \ldots, n$. Search for the non-dominated solutions and

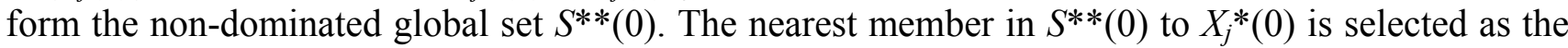
global best $X_{j}^{* *(0)}$ of the $j^{\text {th }}$ particle. Now, set the external set to $S^{* *}(0)$ and update the initial value of the inertia weight $w(0)$.

Step 2: Update the time counter $t=t+1$,

Step 3: Update the inertia weight $w(\mathrm{t})=\alpha w(t-1)$,

Step 4: Using the local best $X_{j}^{*}(t)$ and the global best $X_{j}^{* *}(t)$ of each particle, $j=1, \ldots, n$, the $j^{\text {th }}$ particle velocity in the $k^{\text {th }}$ dimension is updated based on Eq. (23),

Step 5: Based on the updated velocities, each particle changes its position according to the Eq. (22), If a particle violates its position limits in any dimension, set its position at the proper limit,

Step 6: Update the position of the $j^{\text {th }}$ particle by adding to $S_{j}^{*}(\mathrm{t})$. Truncate the dominated solutions in $S_{j}^{*}(\mathrm{t})$ and update them, accordingly, If the size of $S_{j} *(\mathrm{t})$ exceeds a pre-specified value, reduce the size to its maximum limit using the hierarchical clustering algorithm,

Step 7: Form the union of all non-dominated local sets and the non-dominated solutions, where the union includes members in the non-dominated global set $S^{* *}(t)$, Reduce the size by hierarchical clustering algorithm if it exceeds a pre-specified value,

Step 8: Update the external Pareto-optimal set as follows, (a) Copy the members of $S^{* *}(\mathrm{t})$ to the external Pareto set, 
(b) Search the external Pareto set for the non-dominated individuals and eliminate all dominated solutions from the set,

(c) Reduce the set by means of clustering, if the number of the individuals externally stored in the Pareto set exceeds the maximum size,

Step 9: Measure the individual distances between members in $S_{j}^{*}(t)$, and members in $S^{* *}(t)$ in the objective space. If $X_{j}^{*}(t)$ and $X_{j}^{* *}(t)$ are the members of $S_{j}^{*}(t)$ and $S^{* *}(t)$, respectively, which give the minimum distance, Select them as the local best and the global best of the $j^{\text {th }}$ particle, respectively,

Step 10: Stop, if the number of iterations exceeds the maximum then stop, otherwise go to step 2.

Fig. 1 shows details of the proposed MPPSO.

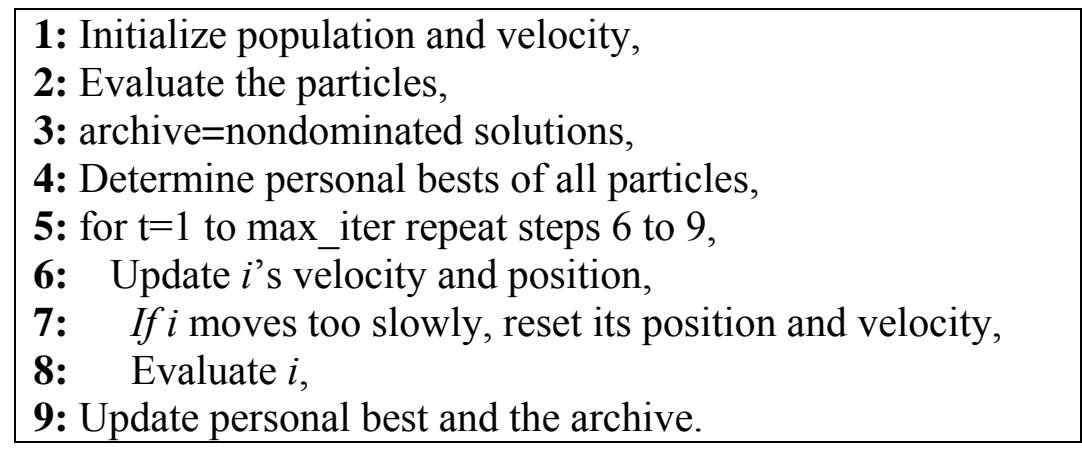

\section{Computational results}

Fig. 1. Pseudo code for MOPSO

We have solved the proposed model of this paper using eight different examples, which are randomly generated. The performance of the proposed model has been compared with an alternative method, NSGA-II. All codes have been coded on MATLAB on a PC computer with Intel ${ }^{\circledR}$ Core ${ }^{\mathrm{TM}}$ Due CPU processor having 2.2 GB RAM.

Table 2

The values of parameters for sample problems

\begin{tabular}{ll}
\hline Parameter & Distribution \\
\hline$H R_{r p t}$ & uniform $(12,18)$ \\
$H G_{g p t}$ & uniform $(18,25)$ \\
$H W_{g w t}$ & uniform $(18,20)$ \\
$T R_{r s p t}$ & uniform $(5,10)$ \\
$T G_{g p w t}$ & uniform $(5,15)$ \\
$T W_{g w z t}$ & uniform $(6,12)$ \\
$F_{g p t}$ & uniform $(750,1500)$ \\
$\alpha_{r g}$ & uniform $(1,3)$ \\
$\widetilde{S C}_{r s t}$ & uniform $(8,12)$ \\
$\widetilde{P C}_{g p t}$ & uniform $(15,20)$ \\
$\widetilde{t i}_{g w z t}$ & uniform $(2,9)$ \\
\hline
\end{tabular}

One of the most important issues in evaluating the performance of an algorithm is to have appropriate attributes. In this paper, we choose three attributes to compare the performance of the proposed model with the performance of the alternative NSGA-II, which are the number of Pareto-optimal solutions 
(NPS), distance (SM) and the diversity of non-dominated (DM) solutions. The second attributes is measured using the following,

$$
S=\left[\frac{1}{n-1} \sum_{i=1}^{n}\left(\bar{d}-d_{i}\right)^{2}\right]^{\frac{1}{2}}
$$

where $d_{i}$ is the distance to $i^{\text {th }}$ solution in non-dominated solutions, $n$ is the number of non-dominated solutions and $\bar{d}$ is the average for $d_{i}$ and the smaller, the better. Diversity is calculated as follows,

$D=\sqrt{\sum_{i=1}^{n} \max \left(x_{i}^{\prime}-y_{i}^{\prime}\right)}$,

where $x_{i}^{\prime}-y_{i}^{\prime}$ is the Euclidian norm between non-dominated solution $x_{i}^{\prime}$ and $y_{i}^{\prime}$. Table 3 and Table 4 summarize the near-optimal solutions for the proposed MOPSO and NSGA-II.

Table 3

The results of the proposed model

\begin{tabular}{lcccccccc}
\hline Problem & $P$ & $S$ & $W$ & $Z$ & NPS & SM & DM & CPU time \\
\hline 1 & 3 & 2 & 2 & 2 & 7 & 0.022 & 0.736 & 7.83 \\
2 & 3 & 2 & 4 & 6 & 5.66 & 0.023 & 0.667 & 9.49 \\
3 & 5 & 3 & 4 & 6 & 6 & 0.010 & 1.058 & 10.36 \\
4 & 5 & 3 & 6 & 8 & 7 & 0.004 & 0.798 & 11.86 \\
5 & 10 & 4 & 6 & 8 & 8.33 & 0.005 & 0.589 & 19.21 \\
6 & 10 & 4 & 8 & 10 & 6.66 & 0.085 & 0.593 & 23.69 \\
7 & 20 & 5 & 8 & 10 & 5 & 0.004 & 0.601 & 32.20 \\
8 & 20 & 5 & 10 & 12 & 7 & 0.004 & 0.685 & 37.17 \\
\hline
\end{tabular}

\section{Table 4}

The results of NSGA-II

\begin{tabular}{lcccccccc}
\hline Problem & $P$ & $S$ & $W$ & $Z$ & NPS & SM & DM & CPU time \\
\hline 1 & 3 & 2 & 2 & 2 & 4.6 & 0.006 & 1.116 & 55.88 \\
2 & 3 & 2 & 4 & 6 & 4.4 & 0.020 & 0.634 & 56.56 \\
3 & 5 & 3 & 4 & 6 & 7 & 0.003 & 0.647 & 57.09 \\
4 & 5 & 3 & 6 & 8 & 5.8 & 0.002 & 0.622 & 58.64 \\
5 & 10 & 4 & 6 & 8 & 4.6 & 0.002 & 0.667 & 62.93 \\
6 & 10 & 4 & 8 & 10 & 4.4 & 0.001 & 0.496 & 64.35 \\
7 & 20 & 5 & 8 & 10 & 5 & 0.001 & 0.405 & 69.68 \\
8 & 20 & 5 & 10 & 12 & 5.4 & 0.001 & 0.449 & 71.12 \\
\hline
\end{tabular}

Table 5 demonstrates the maximum and the minimum amounts for attributes and Table 6 shows the average numbers of solutions found for two methods, which indicates that MOPSO presents better performance compared with NSGA-II. It is also clear that the proposed method of the paper needs significantly less amount of time than alternative method.

Table 5

The maximum and minimum numbers for different attributes and CPU time in sec

\begin{tabular}{|c|c|c|c|c|c|c|c|c|}
\hline \multirow{2}{*}{ Method } & \multicolumn{2}{|c|}{ NPS } & \multicolumn{2}{|c|}{ SM } & \multicolumn{2}{|c|}{$\mathrm{DM}$} & \multicolumn{2}{|c|}{ CPU time } \\
\hline & $\max$ & $\min$ & $\max$ & $\min$ & $\max$ & $\min$ & $\max$ & Min \\
\hline MOPSO & 8.33 & 5 & 0.085 & 0.003 & 1.075 & 0.589 & 59.35 & 7.83 \\
\hline NSGA-II & 7.2 & 4.4 & 0.02 & 0 & 1.116 & 0.284 & 71.49 & 55.88 \\
\hline
\end{tabular}




\section{Table 6}

The average numbers for two algorithms

\begin{tabular}{llllc}
\hline & NPS & SM & DM & CPU time \\
\hline MOPSO & 6.77 & 0.012 & 0.793 & 23.40 \\
NSGA-II & 5.72 & 0.002 & 0.564 & 62.61 \\
\hline
\end{tabular}

Fig. 2 demonstrates the performance of the proposed model in terms of different attributes. As we can observe from the results, the proposed model relatively performs better than the alternative method for some test problems.
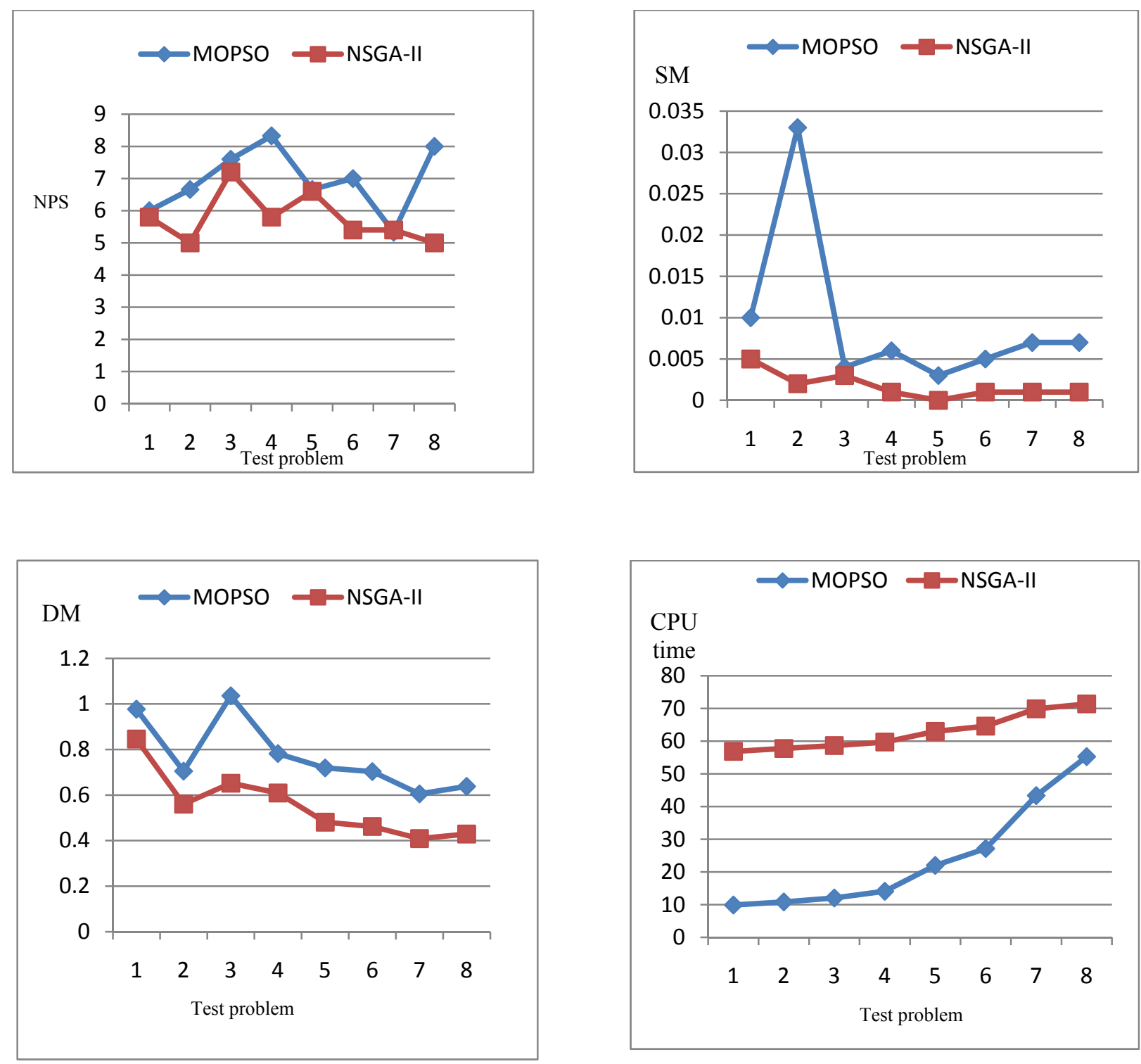

Fig. 2. The performance of the MOPSO vs. NSGA-II

\section{Conclusion}

In this paper, we have presented an integrated supply chain in a form of multi-objective decisionmaking problem. The proposed model of the paper studied different parameters with uncertainty using trapezoid numbers. We first implemented a ranking method to covert the fuzzy model into a crisp one and using multi-objective particle swarm optimization, the resulted model has been solved. 
The results have been compared with the performance of NSGA-II for some benchmark problems, which was promising in terms of different attributes as well as CPU time. As future study, it would be a good idea to compare the performance of the proposed model of this paper with some other techniques such as Niched Pareto genetic algorithm (NPGA), strength Pareto evolutionary algorithm (SPEA) and multi- objective simulated annealing (MOSA) and we leave it for interested researchers for future studies.

\section{Acknowledgment}

The authors would like to thank the anonymous referees for the comments on earlier version of this work, which helped us improve the quality of the paper.

\section{References}

Abido, M.A. (2007). Two-level of nondominated solutions approach to multiobjective particle swarm optimization. In: Proceedings of the 2007 genetic and evolutionary computation conference, GECCO’ 2007, 7-11 July 2007, London, UK, 726-733.

Aliev, R.A., Fazlollahi, B., Guirimov, B.G., \& Aliev, R.R.(2007). Fuzzy-genetic approach to aggregate production-distribution planning in supply chain management. Information Sciences, $177,4241-4255$.

Bilgen, B. (2010). Application of fuzzy mathematical programming approach to the production allocation and distribution supply chain network problem. Expert system with applications, 37, 4488-4495.

Chen, S.P., \& Chang, P.C.(2006). A mathematical programming approach to supply chain models with fuzzy parameters. Engineering Optimization, 38, 647-669.

Coello Coello, C.A., \& Lechuga, M.S. (2002). MOPSO: A proposal for multiple objective particle swarm optimization. In: Proceedings of the 2002 congress on evolutionary computation, 2, 10511056.

Davis, T. (1993). Effective supply chain management. Sloan Management Review, 34, 35-46.

Dehbari, S., Pourrousta, A., Ebrahim Neghad, S., Tavakkoli-Moghaddam, R., \& Javanshir, H.(2012). A new supply chain management method with one-way time window: A hybrid PSO-SA approach. International Journal of Industrial Engineering Computations,3(2), 241-252.

Dubois, D., Fargier, H., \& Fortemps, P. (2003). Fuzzy scheduling: modelling flexible constraints vs. coping with incomplete knowledge. European Journal of Operational Research, 147, 231-252.

Jayaraman, V., \& Ross, A. (2003). A simulated annealing methodology to distribution network design and management. European Journal of Operational Research, 144, 629-645.

Kennedy, J., \& Eberhart, R.C. (1995) Particle swarm optimization. In:Proceedings of the 1995 IEEE International Conference on Neural Networks 4, 1942-1948.

Liang, T.F. (2008). Fuzzy multi-objective production/distribution planning decisions with multiproduct and multi-time period in a supply chain. Computers \& Industrial Engineering, 55(3), 676694.

Liang, T.F.(2008). Integrating production-transportation planning decision with fuzzy multiple goals in supply chains. International Journal of Production Research, 46, 1477-1494.

Liang, T.F., Cheng, \& H.W. (2009). Application of fuzzy sets to manufacturing/distribution planning decisions with multi-product and multi-time period in supply chains. Expert Systems with Applications, 36, 3367-3377.

Moore, J., \& Chapman, R. (1999). Application of particle swarm to multi objective optimization, Department of Computer Science and Software Engineering, Auburn University.

McDonald, C.M., \& Karimi, I.A.(1997). Planning and scheduling of parallel semi-continuous processes. Industrial \& Engineering Chemical Research, 36, 2691-2700. 
Mula, J., Peidro, D., \& Poler, R. (2010). The effectiveness of a fuzzy mathematical programming approach for supply chain production planning with fuzzy demand. International Journal of Production Economics, 128, 136-143.

Peidro, D., Mula, J., Poler, R., \& Verdegay, J.L. (2009). Fuzzy optimization for supply chain planning under supply, demand, and process uncertainties. Fuzzy Sets and Systems, 160, 26402657.

Peidro, D., Mula, J., Jimenez, M., \& Botela, M.D.M. (2010). A fuzzy linear programming based approach for tactical supply chain planning in an uncertainty environment. European Journal of Operational Research, 205, 65-80.

Simchi-Levi, D., Kaminsky, P., \& Simchi-Levi, E. (2000). Designing and Managing the Supply Chain: Concepts, Strategies, and Case Studies. McGraw-Hill, New York.

Sabri, E.H., \& Beamon, B.N. (2000). A multi-objective approach to simultaneous strategic and operational planning in supply chain design. Omega, 28, 581-598.

Syarif, N., Yun, Y., \& Gen, M. (2002). Study on multi-stage logistic chain network: a spanning tree based genetic algorithm approach. Computers \& Industrial Engineering, 43, 299-314.

Torabi, S.A., \& Hassini, E. (2008). An interactive possibilistic programming approach for multiple objective supply chain master planning. Fuzzy Sets and Systems, 159, 193-214.

Yao, J.S., \& Wu, K.(2000). Ranking of fuzzy numbers based on decomposition principle and signed distanced. Fuzzy Sets \& Systems, 116, 275-288.

Zhou, G., Min, H., \& Gen, M. (2002). The balanced allocation of customers to multiple distribution centers in the supply chain network: a genetic algorithm approach. Computers \& Industrial Engineering, 43, 251-261. 